\title{
Dynamic Contrast-Enhanced MRI to Differentiate Parotid Neoplasms Using Golden-Angle Radial Sparse Parallel Imaging
}

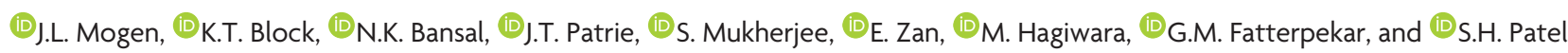

\begin{abstract}
BACKGROUND AND PURPOSE: Conventional imaging frequently shows overlapping features between benign and malignant parotid neoplasms. We investigated dynamic contrast-enhanced MR imaging using golden-angle radial sparse parallel imaging in differentiating parotid neoplasms.
\end{abstract}

MATERIALS AND METHODS: For this retrospective study, 41 consecutive parotid neoplasms were imaged with dynamic contrastenhanced MR imaging with golden-angle radial sparse parallel imaging using 1-mm in-plane resolution. The temporal resolution was 3.4 seconds for 78.2 seconds and 8.8 seconds for the remaining acquisition. Three readers retrospectively and independently created and classified time-intensity curves as follows: 1) continuous wash-in; 2) rapid wash-in, subsequent plateau; and 3) rapid wash-in with washout. Additionally, time-intensity curve-derived semiquantitative metrics normalized to the ipsilateral common carotid artery were recorded. Diagnostic performance for the prediction of neoplasm type and malignancy was assessed. Subset multivariate analysis $(n=32)$ combined semiquantitative time-intensity curve metrics with ADC values.

RESULTS: Independent time-intensity curve classification of the 41 neoplasms produced moderate-to-substantial interreader agreement $(\kappa=0.50-0.79)$. The time-intensity curve classification threshold of $\geq 2$ predicted malignancy with a positive predictive value of $56.0 \%$ $66.7 \%$, and a negative predictive value of $92.0 \%-100 \%$. The time-intensity curve classification threshold of $<2$ predicted pleomorphic adenoma with a positive predictive value of $87.0 \%-95.0 \%$ and a negative predictive value of $76.0 \%-95.0 \%$. For all readers, type 2 and 3 curves were associated with malignant neoplasms $(P<.001)$, and type 1 curves, with pleomorphic adenomas $(P<.001)$. Semiquantitative analysis for malignancy prediction yielded an area under the receiver operating characteristic curve of $0.85(95 \% \mathrm{Cl}, 0.73-0.99)$. Combining time-to-maximum and ADC predicts pleomorphic adenoma better than either metric alone $(P<.001)$.

CONCLUSIONS: Golden-angle radial sparse parallel MR imaging allows high spatial and temporal resolution permeability characterization of parotid neoplasms, with a high negative predictive value for malignancy prediction. Combining time-to-maximum and ADC improves pleomorphic adenoma prediction compared with either metric alone.

ABBREVIATIONS: $A U C=$ area under the curve; $\mathrm{DCE}=$ dynamic contrast-enhanced; $\mathrm{GRASP}=$ golden-angle radial sparse parallel; NPV $=$ negative predictive value; PPV = positive predictive value; $\mathrm{ROC}=$ receiver operating characteristic; SImax = maximum signal intensity; TIC = time-intensity curve; Tmax = time-to-maximum

$\mathbf{P}$ arotid neoplasms include a variety of benign and malignant lesions. ${ }^{1}$ While most parotid neoplasms are benign pleomorphic adenomas and are often treated with superficial parotidec-

Received October 15, 2018; accepted after revision March 31, 2019.

From the Department of Radiology (.L.M.), Tufts Medical Center, Boston, Massachusetts; Department of Radiology (K.T.B., N.K.B., E.Z., M.H., G.M.F.), New York University Langone Medical Center, New York, New York; Division of Biostatistics and Epidemiology (J.T.P.), University of Virginia, Charlottesville, Virginia; and Department of Radiology and Medical Imaging (S.M., S.H.P.), University of Virginia Health System, Charlottesville, Virginia.

Preliminary results from this subject group previously presented at: American Society of Neuroradiology Annual Meeting and the Foundation of the ASNR Symposium, April 22-27, 2017; Long Beach, California.

Please address correspondence to Sohil H. Patel, MD, Department of Radiology and Medical Imaging, University of Virginia Health System, PO Box 800170, Charlottesville, VA 22908; e-mail: shp4@virginia.edu tomy (or in some instances, conservative monitoring), differentiating such lesions from those considered at high risk for malignancy can be useful for management because malignant parotid neoplasms are more typically scheduled for total parotidectomy with possible facial nerve sacrifice. ${ }^{2-4}$ Clinical examination and conventional MR imaging sequences demonstrate considerable overlap among various pathologic entities. Almost all parotid neoplasms demonstrate contrast enhancement and intrinsically low signal on T1WI. ${ }^{5}$ While features including irregular/infiltrative margins, heterogeneous signal intensity, and low signal on T2WI correlate with malignant pathology, ${ }^{2,5}$ the performance of

Indicates article with supplemental on-line tables.

http://dx.doi.org/10.3174/ajnr.A6055

AJNR Am J Neuroradiol 40:1029-36 Jun 2019 www.ajnr.org 


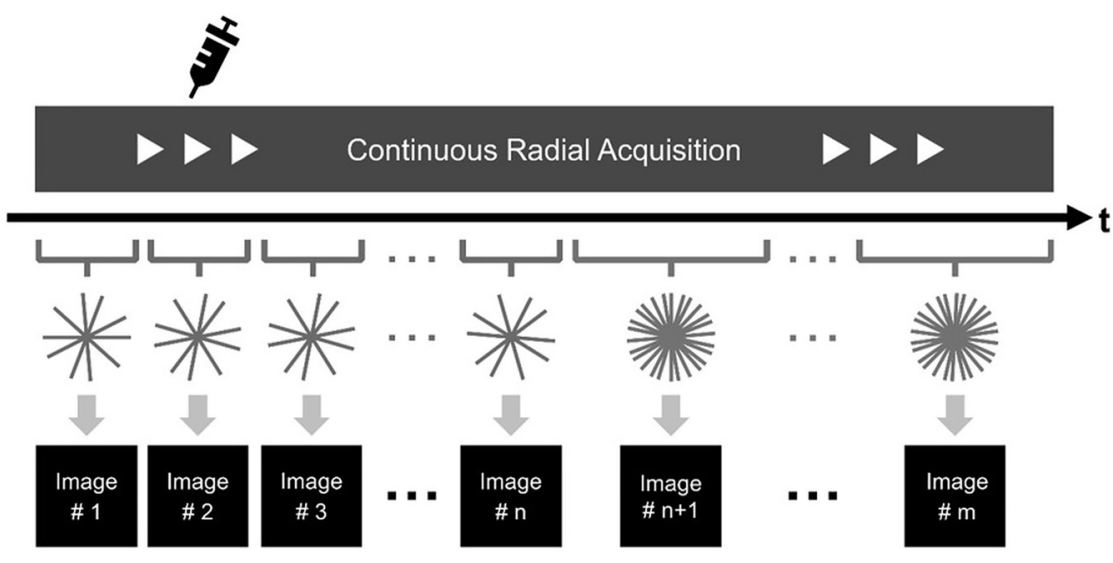

FIG 1. GRASP uses a continuous radial stack-of-stars acquisition of $k$-space during which the contrast agent is injected (20-second injection delay). Due to use of the golden-angle ordering scheme, an arbitrary number of consecutive frames can be combined into a single image frame and a desired temporal resolution can be thus assigned. Because the $k$-space data for each frame are highly undersampled, GRASP uses a compressed-sensing reconstruction with temporal totalvariation constraint. Additionally, by combining all acquired frames into a single image frame, one can acquire high-resolution fat-suppressed T1-weighted anatomic images of the entire neck with an excellent signal-to-noise ratio (Figs 2 and 5 for examples).

conventional imaging reported in the literature has been variable. One study evaluating 98 parotid neoplasms found that conventional imaging was only $50 \%$ sensitive for the presence of malignancy. ${ }^{6}$ Another study provided more encouraging results with a specificity of $95 \%$ for pleomorphic adenoma based on a combination of conventional features, though only 21 of the 41 pleomorphic adenomas included in this study met the proposed criteria and the overall diagnostic accuracy was $74.8 \% .{ }^{7}$ Preoperative biopsy represents a useful diagnostic tool for parotid masses. However, potential limitations include seeding of adjacent tissues after capsular violation, small tissue volumes for pathologic analysis, and procedural risks that include facial nerve injury, sialocele formation, or fistula formation. ${ }^{2,8,9}$

Golden-angle radial sparse parallel (GRASP) MR imaging is a 3D gradient recalled-echo dynamic contrast-enhanced (DCE) MR imaging technique using both compressed sensing and parallel imaging to accelerate data acquisition. GRASP MR imaging uses a radial "spoke-wheel" method of $k$-space sampling in the xy plane according to the golden-angle scheme and Cartesian sampling in the $\mathrm{z}$ axis. ${ }^{10}$ Acquiring radial data according to the golden-angle ordering scheme allows dynamic imaging data acquisition as a single continuous scan. Dynamic image reconstruction is achieved by grouping the data into sequential timeframes at a desired temporal resolution (as fast as 2.5 seconds per frame). Iterative image reconstruction with temporal total-variation constraint permits both high temporal and spatial resolution. Thus, GRASP imaging provides both high-spatial-resolution anatomic imaging of a desired imaging volume and also hightemporal-resolution DCE-MR imaging data from the same image acquisition. Prior work in abdominal, breast, and other head and neck applications confirms the excellent image quality, improved fat suppression, and reduced motion and pulsation artifacts of GRASP-derived images compared with conventional anatomic MR imaging techniques, with the additional benefit of concurrently acquired DCE-MR imaging data. ${ }^{11-17}$
The purpose of this study was to investigate the accuracy of qualitative and semiquantitative metrics derived from DCE-MR imaging using GRASP imaging to differentiate parotid neoplasms. Unlike most prior studies, we evaluated semiquantitative metrics normalized for an internal normal reference standard, and we additionally performed comprehensive assessment of interreader reliability of qualitative GRASP-derived DCE analysis, which can be easily integrated into day-today workflow. The novel ability of GRASP to provide high-resolution anatomic imaging concurrently with DCE-MR imaging gives a further rationale for investigating GRASP imaging in this context. Finally, the added value of DWI was assessed in a subset cohort.

\section{MATERIALS AND METHODS Patient Selection}

This retrospective study of patient data received New York University Langone Medical Center institutional review board approval, and the requirement for informed consent was waived. Health Insurance Portability and Accountability Act-compliant techniques for data management and analysis were used.

From July 2015 through August 2016, DCE-MR imaging using the GRASP technique was performed on 52 consecutive parotid lesions in 48 patients. The patient mean age was 59.8 years with a range of 29-85 years. There were 16 men and 21 women. Eleven patients with 11 parotid lesions were excluded due to the following: a lack of a pathologic diagnosis $(n=9)$, nondiagnostic examination due to poor contrast bolus quality $(n=1)$, and a purely cystic lesion $(n=1)$. A total of 41 parotid lesions in 37 patients were ultimately included. In all cases, the final diagnosis was determined on the basis of histopathologic analysis following surgical resection.

\section{GRASP DCE-MR Imaging Technique}

A detailed description of DCE-MR imaging based on the GRASP technique has been published previously. ${ }^{10}$ In brief, the sequence entails a continuous radial $k$-space acquisition according to the golden-angle $\left(111.25^{\circ}\right)$ scheme to obtain a dynamic acquisition of a desired length, during which a contrast agent is injected (Fig 1). After data acquisition is complete, consecutive spokes are grouped into discrete temporal frames. The number of spokes combined into each frame can be chosen arbitrarily. For a low number of spokes/frame (as needed to achieve high imaging speed), the data are highly undersampled. Therefore, both parallel imaging and compressed sensing are applied in the reconstruction process to obtain artifact-free dynamic images with both high spatial and high temporal resolution (the compressed-sensing implementation uses a temporal total variation constraint). The reconstructed images were then analyzed as described below. 
Patients in this study were scanned on both $1.5 \mathrm{~T}$ and 3T MR imaging units. Dynamic imaging had 144 axial sections at a $2-\mathrm{mm}$ slice thickness. The FOV was $256 \times 256 \mathrm{~mm}$ at a matrix size of $256 \times$ 256 , resulting in a $1-\mathrm{mm}$ in-plane resolution. Anatomic coverage comprised the whole neck from the superior orbit to sternal notch. TR and TE were 4.3 and $2.1 \mathrm{~ms}$, respectively, with a $12^{\circ}$ flip angle. Temporal resolution was $3.4 \mathrm{~s} /$ frame for the first 78.2 seconds and 8.8 $\mathrm{s} /$ frame through the remainder of the examination for a total dynamic acquisition of 240 seconds. A single dose of gadobutrol (0.1 $\mathrm{mL} / \mathrm{kg}$, Gadavist; Bayer Schering Pharma, Berlin, Germany) was administered intravenously at a rate of $3 \mathrm{~mL} / \mathrm{s}$ with an injection delay of 20 seconds relative to the start of the sequence.

\section{Qualitative Time-Intensity Curve Classification}

Three readers independently postprocessed and analyzed the DCE-MR imaging data for all 41 parotid neoplasms using the software Olea Sphere 3.0 (Olea Medical, La Ciotat, France). Reader A had 5 years of neuroradiology experience, reader $\mathrm{B}$ had 1 year of neuroradiology experience, and reader $\mathrm{C}$ was a radiology resident. The readers were blind to the clinical history, pathologic diagnosis, and all prior and subsequent imaging studies. Each reader was instructed to manually create a single ROI that circumscribed the enhancing component of the neoplasm on the axial image judged to contain the largest enhancing area. Vessels and nonenhancing portions of the lesions (eg, cystic components, centrally necrotic components) were excluded from the ROI. Time-intensity curves (TICs) were created from the assigned ROI, and each reader assigned to each TIC 1 of 3 curve classifications: 1) continuously rising, 2) initial rapid wash-in with subsequent plateau, and 3) initial rapid wash-in with subsequent washout (Fig 2).

\section{Semiquantitative TIC Analyses}

Semiquantitative analysis was undertaken subsequent to the qualitative analysis by 2 of the readers (A and B) in consensus. At the time of semiquantitative analysis, the 2 readers remained blinded to the clinical history, pathologic diagnosis, and all prior and subsequent imaging studies. An ROI was chosen within the parotid neoplasm to create a TIC, as described above. An additional ROI was chosen within the ipsilateral common carotid artery to serve as an internal reference standard. The common carotid artery was chosen over the external carotid artery or more distal branches due to improved interand intraobserver reproducibility of the resulting TICs. TIC metrics including time-to-maximum (Tmax), maximum signal intensity (SImax ), wash-in slope, washout slope, and area under the curve were calculated for the parotid neoplasm and normalized to (ie, divided by) values obtained from the ipsilateral common carotid artery.

\section{Diffusion-Weighted Imaging Data Collection}

A DWI sequence was available in a 32-lesion (28 patient) subset of those enrolled. DWI was performed using EPI with a TR and TE of 7500 and $87 \mathrm{~ms}$, respectively. Matrix size was $128 \times 128$, slice thickness/gap was $4.0 / 4.8 \mathrm{~mm}$, and b-values of 0 and $1000 \mathrm{~s} / \mathrm{mm}^{2}$ were obtained. Three gradient directions were used. An ROI was assigned to match the ROI used in the semiquantitative TIC analysis. Mean ADC values were derived from the assigned ROI.

\section{Statistical Methods}

Categoric data were summarized by frequencies and percentages, and continuous scaled data were summarized by the mean and SD and the range of the empiric distribution.

Qualitative TIC Analysis. Interreader agreement with respect to the TIC classification was evaluated via the unweighted $\kappa$ statistic. Conventional receiver operating characteristic (ROC) analyses were conducted per reader to determine the ROC associated with malignant parotid neoplasm prediction and pleomorphic adenoma prediction as a function of TIC classification threshold. These categories were chosen on the basis of the frequency of pleomorphic adenoma relative to other parotid neoplasms and the importance of determining the malignant status of a lesion before any intervention. Bivariate associations between each reader's qualitative TIC classifications and the lesion malignant parotid neoplasm status (yes, no) and the lesion pleomorphic adenoma status (yes, no) were evaluated via exact logistic regression. The mid- $P$ value method of Clopper and Pearson ${ }^{18}$ was used to derive the $P$ value of the exact test.

Semiquantitative TIC Analyses. Univariate and multivariate relationships between the TIC-derived semiquantitative metrics and the malignant parotid neoplasm status (yes, no) and the pleomorphic adenoma status (yes, no) were evaluated via univariate and multivariate logistic regression, respectively. Conventional ROC analyses were conducted to determine the operator characteristics associated with malignant parotid neoplasms and pleomorphic adenoma prediction as a function of the predicted probabilities derived from the TIC-derived semiquantitative predictor-based logistic regression model.

Subset Analyses of ADC Values and Semiquantitative TIC-Derived Metrics. Using the subset data of the 32 parotid neoplasms that underwent a DWI sequence, univariate and multivariate logistic regression analyses were performed to determine whether the ADC values derived from DWI provide additional diagnostic information that is not provided by the TIC-derived semiquantitative metrics. Type III $\chi^{2}$ tests were used to test whether the addition of prediction information provided by ADC about malignant parotid neoplasm and pleomorphic adenoma is beyond what would be expected by chance.

Statistical Software. The statistical software package SAS, Version 9.4 (SAS Institute, Cary, North Carolina) was used to conduct the qualitative TIC analyses. The statistical software package R, Version 3.4.2 (http://www.r-project.org) was used to conduct the semiquantitative TIC analyses and the subset analysis.

\section{RESULTS}

The mean patient age was $59.8 \pm 14.2$ years with a range of $29-85$ years. There were 16 men $(43.2 \%)$ and 21 women $(56.8 \%)$. There were $27(65.9 \%)$ benign neoplasms (21 pleomorphic adenomas, 4 Warthin tumors, 2 oncocytomas) and 14 (34.1\%) malignant neoplasms ( 2 acinic cell carcinomas, 4 squamous cell carcinomas, 2 adenoid cystic carcinomas, 1 carcinoma ex pleomorphic adenoma, 2 mucoepidermoid carcinomas, 2 salivary duct carcinomas, and 1 spindle cell carcinoma). There was no statistically significant association between patient age or sex and the pathologic diagnosis. 


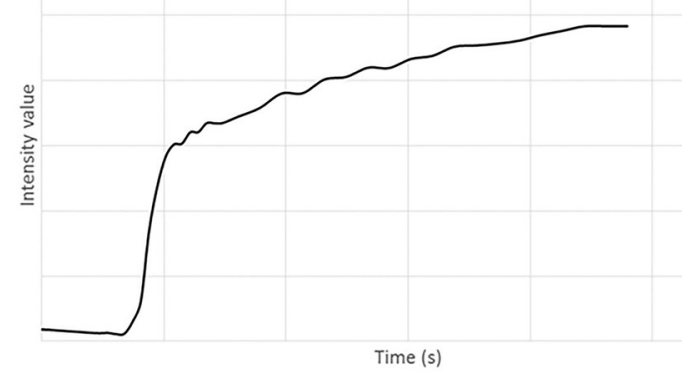

A
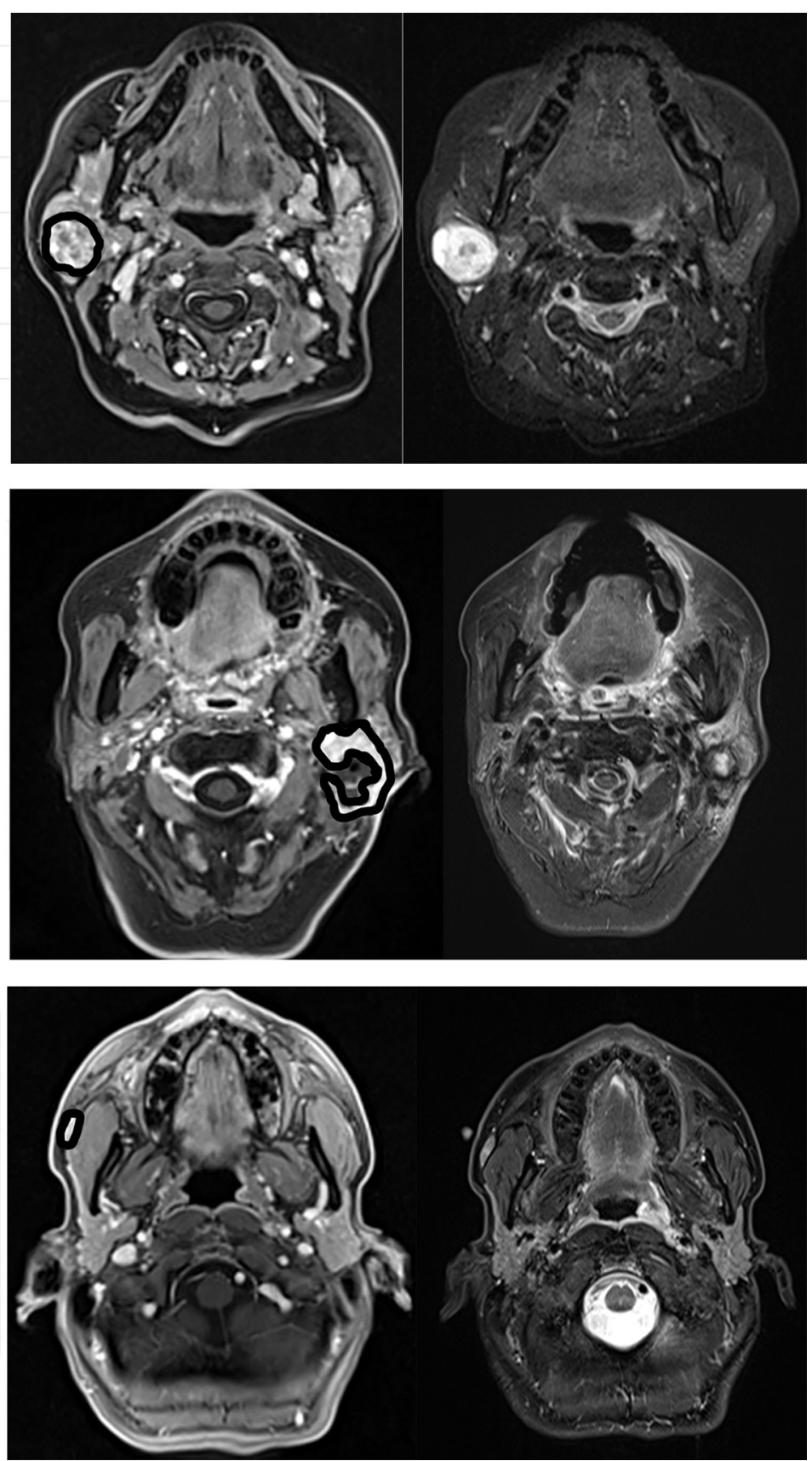

FIG 2. Examples of time-intensity curve morphology for each qualitatively applied category with representative axial contrast-enhanced GRASP and axial T2WI demonstrating the lesions and ROIs used for TIC creation. A, Continuous rise in a patient with pleomorphic adenoma. $B$, Rapid rise with a plateau in a patient with squamous cell carcinoma. C, Wash-in and washout in a patient with myoepithelial carcinoma of the accessory parotid gland. Notice that the GRASP sequence permits high-temporal-resolution dynamic permeability characterization concurrent with high-spatial-resolution fat-suppressed T1-weighted anatomic imaging in a single acquisition.

\section{Qualitative TIC Analysis}

Interreader agreement for the TIC classifications of readers A and B was substantial ( $\kappa=0.79 ; 95 \% \mathrm{CI}, 0.63-0.96)$ and moderate for readers $\mathrm{A}$ and $\mathrm{C}(\kappa=0.54 ; 95 \% \mathrm{CI}, 0.34-0.73)$ and readers $\mathrm{B}$ and $\mathrm{C}(\kappa=0.50 ; 95 \% \mathrm{CI}, 0.30-0.71)$.

After we combined TIC types 2 and 3 , there was a significant association between TIC types 2 and 3 and parotid malignancy for all readers $(P<.001$ for all). At a TIC classification threshold of $>1$ (ie, TIC types 2 and 3 ), the positive predictive value (PPV) for predicting malignancy ranged from $56.0 \%$ to $66.7 \%$ among readers and the negative predictive value (NPV) for predicting malignancy ranged from $91.7 \%$ to $100 \%$. Among malignant lesions, $92.9 \%$ of all reader TIC assignments were type 2 or 3 .

After we combined TIC types 2 and 3, there was a significant association between TIC type 1 and pleomorphic adenoma for all readers $(P<.001$ for all). At a TIC assignment threshold of $<2$ (ie, TIC type 1), the PPV for predicting pleomorphic adenoma ranged from $87.0 \%$ to $95.0 \%$ among readers, and the NPV for predicting pleomorphic adenoma ranged from $76.0 \%$ to $95.0 \%$ among the readers.

The associations between the TIC classification and parotid malignancy and the TIC classification and pleomorphic adenoma 


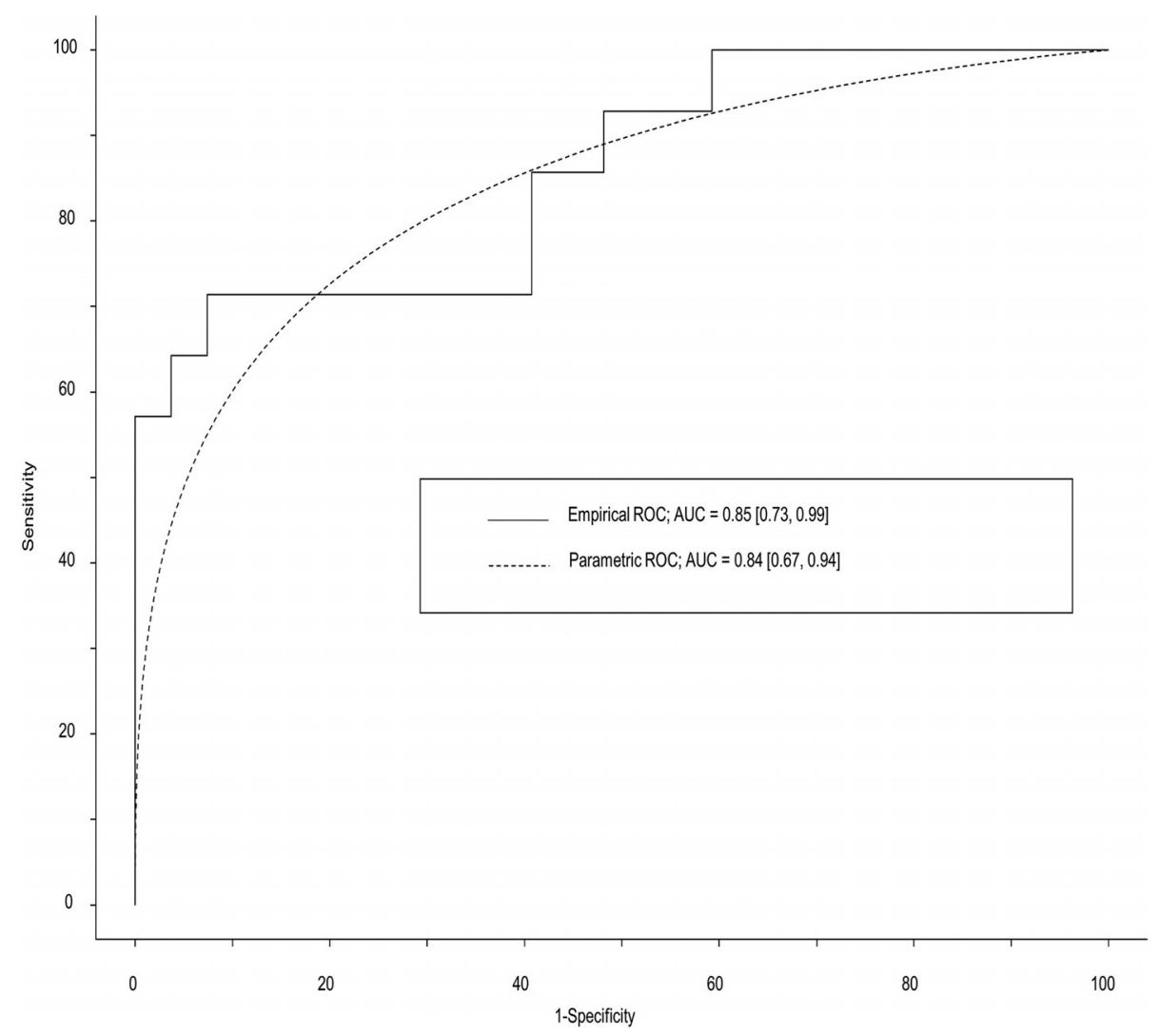

FIG 3. Empiric and binormal parametric ROC curves for discrimination of malignant and nonmalignant parotid neoplasms based on Tmax, AUC, washout slope, and SImax.

are summarized in On-line Table 1. Complete summaries of the ROC analyses are provided in On-line Table 2. Figure 2 shows examples of 3 cases.

Among all reader TICs created for Warthin tumors and oncocytomas, $88.9 \%(n=16)$ were assigned as type 2 or 3 , and $11.1 \%$ $(n=2)$, as type 1 .

\section{Semiquantitative TIC Analyses}

Univariate logistic regression analyses that examined the bivariate associations between parotid malignancy and the TIC semiquantitative metrics (normalized to the ipsilateral common carotid artery) revealed that Tmax (OR, 7.14; 95\% CI, 1.73-29.30; $P=.007)$ and wash-in slope (OR, 2.63; 95\% CI, 1.07-6.46; $P=.034$ ) were significantly associated with parotid malignancy. On the basis of type III $\chi^{2}$ tests, a multivariate logistic regression analysis identified Tmax $(P=$ $.018)$, area under the curve (AUC) $(P=.030)$, washout slope $(P=$ $.031)$, and $\operatorname{SImax}(P=.048)$ as unique predictors of parotid malignancy. ROC curves and diagnostic characteristics for discrimination between nonmalignant and malignant parotid neoplasms based on Tmax, AUC, washout slope, and SImax are shown in Fig 3 (and On-line Table 3). The area under the curve for empiric and parametric ROC curves was 0.85 (95\% CI, 0.73-0.99) and 0.84 (95\% CI, $0.67-0.94)$, respectively.

Univariate logistic regression analysis that examined the bivariate associations between pleomorphic adenoma and TIC semiquantitative metrics (normalized to the ipsilateral common carotid artery) revealed that $\mathrm{Tmax}(\mathrm{OR}, 27.38 ; 95 \% \mathrm{CI}$, 4.48-167.18; $P<.001)$ and wash-in slope (OR, 3.03; 95\% CI,
1.13-8.16; $P=.028)$ were significantly associated with predicting pleomorphic adenoma. On the basis of type III $\chi^{2}$ tests, a multivariate logistic regression analysis identified $\operatorname{Tmax}(P=$ .024) as the only unique predictor of pleomorphic adenoma. ROC curves and diagnostic characteristics for discrimination of nonpleomorphic adenoma and pleomorphic adenoma based on Tmax are shown in Fig 4 (and On-line Table 4). The area under the curve for empiric and parametric ROC curves was 0.87 (95\% CI, 0.73-0.95) and 0.86 (95\% CI, 0.73-0.95), respectively.

\section{Subset Analyses of ADC Values and Semiquantitative TIC-Derived Metrics}

A subset of 32 parotid neoplasms underwent a DWI sequence. The odds ratio for the association between ADC and malignancy was 102.39 (95\% CI, 2.38-4402.38; $P=.016$ ). The odds ratio for the association between ADC and pleomorphic adenoma was 71.95 (95\% CI, 4.74-1091.38; $P=.002$ ).

For the determination of parotid malignancy, a logistic regression model combining Tmax, AUC, washout slope, and SImax with ADC could not be created because the covariance matrix of this model is singular. For the determination of parotid malignancy, a logistic regression model that includes both Tmax and ADC is superior to a model based on Tmax alone ( $\chi^{2}$ statistic, 11.39; $P<.001$ ), but not superior to a model containing ADC alone ( $\chi^{2}$ statistic, $\left.0.04 ; P=.846\right)$.

For the determination of pleomorphic adenoma, a logistic regression model that includes both Tmax and ADC is superior 


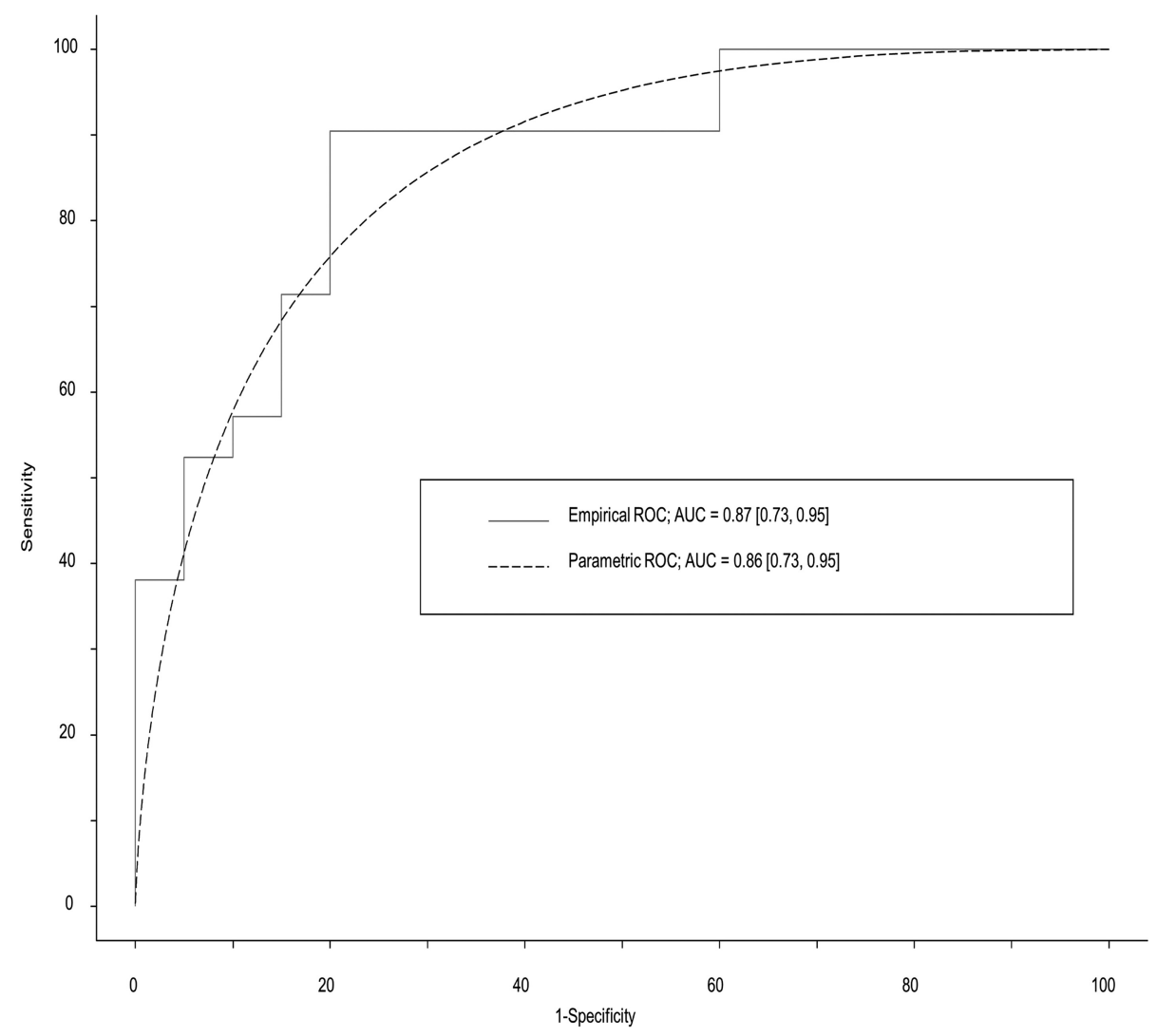

FIG 4. Empiric and binormal parametric ROC curves for discrimination between pleomorphic adenomas and nonpleomorphic adenomas based on Tmax.

to both a model containing Tmax alone ( $\chi^{2}$ statistic, $13.94 ; P<$ $.001)$ and a model containing ADC alone ( $\chi^{2}$ statistic, 12.53 ; $P<.001)$.

Figure 5 shows sample cases incorporating GRASP-derived DCE data and ADC data. All raw data from qualitative and semiquantitative analyses are provided in On-line Table 5.

\section{DISCUSSION}

Our results highlight the technical advantages of GRASP, a sequence that concurrently obtains high spatial resolution and fatsuppressed anatomic imaging of the entire neck as well as permeability information for noninvasive functional characterization of parotid neoplasia. We were able to document the independent processing and interpretation of DCE-MR imaging data in parotid neoplasms by multiple readers, which is a helpful step in attempting to generalize the application of DCE-MR imaging beyond the research setting. To our knowledge, only 1 prior study has evaluated interreader reliability (with 2 readers) for DCE-MR imaging analysis in parotid neoplasms. ${ }^{1}$

The results of our semiquantitative analysis are consistent with previous studies, specifically the findings that pleomorphic adenomas are associated with a relatively long Tmax, while malignant lesions are associated with a shorter Tmax..$^{1,2,6,8}$ Multiple Tmax threshold values are reported in the literature for the prediction of pleomorphic adenoma or malignancy, possibly due to differences in the DCE technique, which limits direct comparison of values across studies. Similar to prior analyses, we found that relying on this 1 parameter for the prediction of specific histologic diagnoses was limited, primarily due to the overlapping TIC features of Warthin tumors and oncocytomas with those of malignant lesions. The most useful way to apply permeability data in clinical practice appears to relate to its high negative predictive value for malignancy. Namely, parotid neoplasms with a long Tmax are unlikely to represent malignant neoplasms. Moreover, our multivariate analysis showed that AUC, washout slope, and SImax were also unique predictors of parotid malignancy. Finally, all of our semiquantitative metrics were normalized to an anatomic reference (common carotid artery). Most prior studies did not normalize their data in this fashion. Reference tissue normalization is important because it provides a method to correct for the numerous sources of variability across different patients and scanners. ${ }^{1,2,6,8,19,20,22-24}$

Most interesting, our subset analysis incorporating ADC values showed that a multivariate model combining ADC values with Tmax showed a significantly improved performance for the prediction of pleomorphic adenoma compared with either parameter alone. With respect to malignancy characterization, previous studies have shown that the combined information of neovascularization (shorter Tmax) and higher cellularity (lower ADC) may help to identify parotid malignancies as distinct from benign neoplasms. ${ }^{20,21}$ While our results did show that ADC combined with Tmax outperformed Tmax alone when predicting malignancy, our analysis here was limited because we were not able to combine ADC with the full logistic regression model that included Tmax, AUC, washout slope, and SImax. 

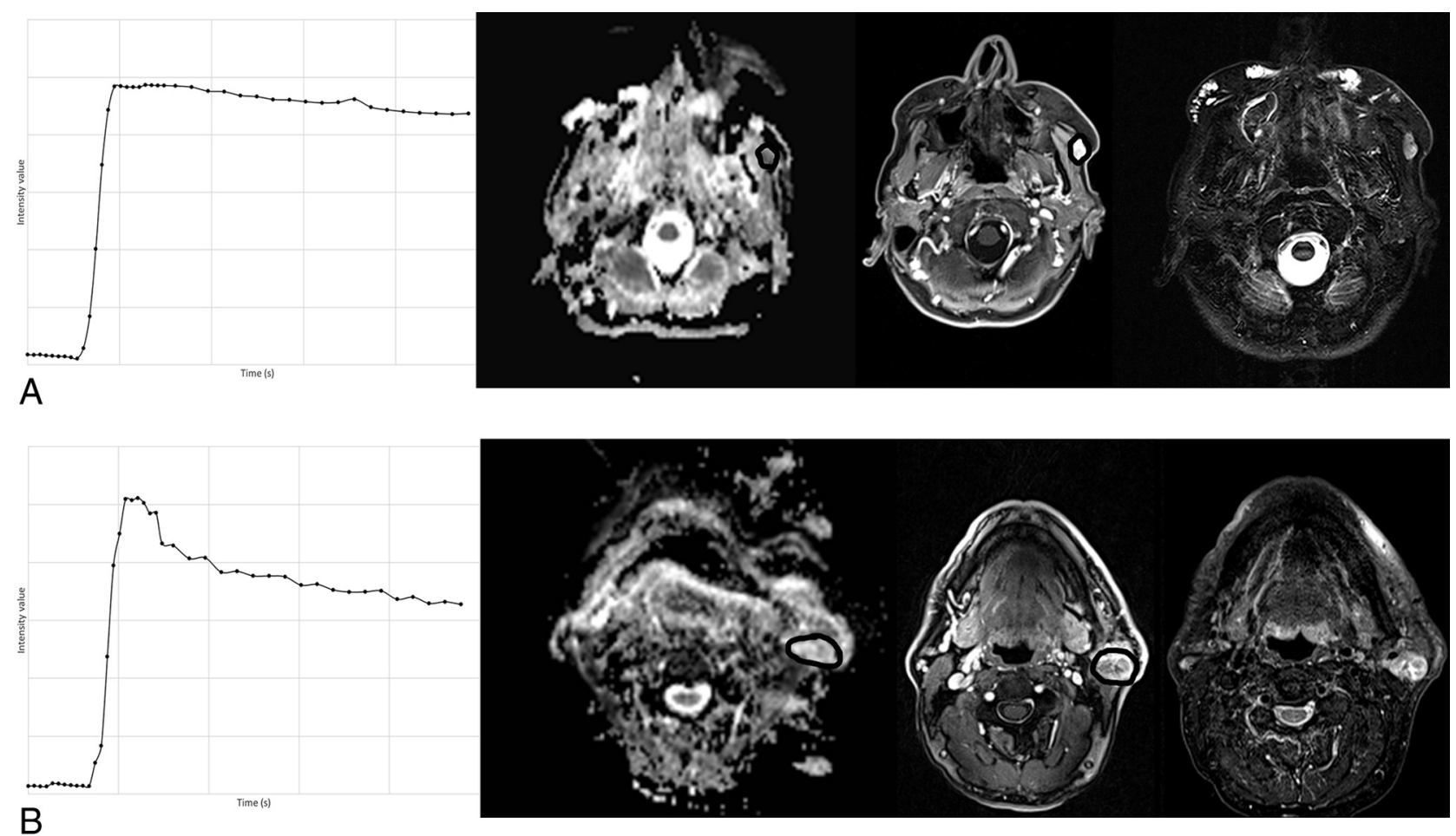

FIG 5. Representative examples of TICs, ADC maps, contrast-enhanced GRASP, and T2WI. A, Acinic cell carcinoma, displaying a type $2 / 3$ curve TIC and a relatively low ADC of 926, which both favor a malignant neoplasm. B, Warthin tumor, displaying a characteristic type 3 TIC in combination with a relatively elevated ADC of 1616. The oncocytomas in our cohort had a similar combination of TIC and ADC features, whereas nearly all pleomorphic adenomas had type 1 TICs in combination with relatively elevated ADC values.

While several of our results support the results of prior literature, the GRASP technique has several key features that represent an improvement over techniques used in prior studies of DCE-MR imaging in parotid neoplasia. Uniquely, GRASP simultaneously provides high-spatial-resolution anatomic T1-weighted images of large anatomic coverage (eg, entire neck) with dynamic high-temporal-resolution permeability imaging of the entire imaging volume. GRASP uses a continuous radial stack-of-stars acquisition of $k$-space, and an arbitrary number of consecutive radial spokes can be combined into a single image frame to create DCE imaging of a desired temporal resolution. Additionally, by combining all spokes into a single image frame, one can create high-resolution fat-suppressed anatomic images of the entire neck with an excellent signal-to-noise ratio. Thus, GRASP can be routinely integrated into a parotid gland imaging protocol as a diagnostic anatomic sequence without using examination time solely to obtain permeability information. Although some authors have performed DCE-MR imaging at a similar temporal resolution, the techniques used in those studies were often limited to either a single slice or a smaller portion of the volume of interest at a lower spatial resolution. ${ }^{6,22,23}$ Because GRASP acquires high-temporal-resolution permeability imaging for entire neck coverage, it has an added benefit of ensuring that a useful reference standard (such as the common carotid artery) is included in the imaging volume for normalizing imaging metrics. It also removes the possibility of inadvertently excluding portions of parotid neoplasms when selecting the imaging subvolume for DCE acquisition. Finally, a distinct advantage to radial $k$-space sampling is its substantially reduced sensitivity to motion arti- facts. In fact, none of the cases in our study required exclusion due to motion artifacts. ${ }^{10-12}$

One limitation of our study is our cohort size $(n=41)$, and we specifically note here the important 2016 study by Yuan et al, ${ }^{25}$ which included DCE imaging in a large cohort of parotid neoplasms. However, despite our smaller patient cohort, we believe our study remains of interest, predominantly due to the application of the GRASP imaging technique as discussed above. Specifically, our GRASP technique enabled entire neck coverage with 2-mm slice thickness (compared with $5 \mathrm{~mm}$ in the Yuan study) despite a similar reported temporal resolution. Moreover, Yuan et al did not normalize their DCE metrics using an anatomic reference as we did. Aside from such technical differences, we performed a comprehensive assessment of interreader reliability for independently processing and categorizing the parotid lesion TIC using DCE-MR imaging, whereas Yuan et al used a consensus reading.

Additional limitations of our study include its retrospective design. We undertook qualitative and semiquantitative methods of analysis rather than "quantitative" analysis using baseline T1mapping that allows determination of parameters such as the volume transfer constant between the extravascular extracellular space and plasma or extravascular extracellular space volume per tissue volume. ${ }^{26}$ However, such quantitative techniques are themselves limited by the application of various physiologic assumptions across different patients. We included MR imaging examinations from both $1.5 \mathrm{~T}$ and $3 \mathrm{~T}$ scanners and acknowledge that different scanners might yield differing DCE data. Finally, the 
GRASP sequence is currently limited to a single vendor and thus not yet available to all MR imaging sites for routine clinical use.

\section{CONCLUSIONS}

GRASP-derived DCE-MR imaging for parotid neoplasms introduces several technical advantages, including the ability to create high-temporal-resolution dynamic data for permeability assessment concurrent with high-spatial-resolution, large-anatomiccoverage, diagnostic imaging of the whole neck. GRASP-derived DCE-MR imaging achieves a high NPV when determining the presence of parotid malignancy and excellent diagnostic performance in predicting pleomorphic adenomas.

Disclosures: Kai Tobias Block-UNRELATED: Patents (Planned, Pending or Issued): New York University GRASP patent, Comments: New York University owns the patent on the GRASP technique of which I am a coinventor. No royalties have been paid.* Sohil H. Patel—UNRELATED: Grants/Grants Pending: Radiological Society of North America Research Scholar Grant.* Elcin Zan-UNRELATED: Grants/Grants Pending: Novartis/Advanced Accelerator Applications, Comments: Clinical trial funding is pending. * *Money paid to the institution.

\section{REFERENCES}

1. Lechner Goyault J, Riehm S, Neuville A, et al. Interest of diffusionweighted and gadolinium-enhanced dynamic MR sequences for the diagnosis of parotid gland tumors. J Neuroradiol 2011;38:77-89 CrossRef Medline

2. Yabuuchi H, Fukuya T, Tajima T, et al. Salivary gland tumors: diagnostic value of gadolinium-enhanced dynamic MR imaging with histopathologic correlation. Radiology 2003;226:345-54 CrossRef Medline

3. Valentini V, Fabiani F, Perugini M, et al. Surgical techniques in the treatment of pleomorphic adenoma of the parotid gland: our experience and review of literature. J Craniofac Surg 2001;12:565-68 CrossRef Medline

4. Abdel Razek AA, Samir S, Ashmalla GA. Characterization of parotid tumors with dynamic susceptibility contrast perfusion-weighted magnetic resonance imaging and diffusion-weighted MR imaging. J Comput Assist Tomogr 2017;41:131-36 CrossRef Medline

5. Yousem DM, Kraut MA, Chalian AA. Major salivary gland imaging. Radiology 2000;216:19-29 CrossRef Medline

6. Lam PD, Kuribayashi A, Imaizumi A, et al. Differentiating benign and malignant salivary gland tumours: diagnostic criteria and the accuracy of dynamic contrast-enhanced MRI with high temporal resolution. Br J Radiol 2015;88:20140685 CrossRef Medline

7. Zaghi S, Hendizadeh L, Hung T, et al. MRI criteria for the diagnosis of pleomorphic adenoma: a validation study. Am J Otolaryngol 2014; 35:713-18 CrossRef Medline

8. Hisatomi M, Asaumi J, Yanagi Y, et al. Diagnostic value of dynamic contrast-enhanced MRI in the salivary gland tumors. Oral Oncol 2007;43:940-47 CrossRef Medline

9. Haldar S, Sinnott JD, Tekeli KM, et al. Biopsy of parotid masses: review of current techniques. World J Radiol 2016;8:501-05 CrossRef Medline

10. Feng L, Grimm R, Block KT, et al. Golden-angle radial sparse parallel MRI: combination of compressed sensing, parallel imaging, and golden-angle radial sampling for fast and flexible dynamic volumetric MRI. Magn Reson Med 2014;72:707-17 CrossRef Medline

11. Chandarana H, Feng L, Block TK, et al. Free-breathing contrast- enhanced multiphase MRI of the liver using a combination of compressed sensing, parallel imaging, and golden-angle radial sampling. Invest Radiol 2013;48:10-16 CrossRef Medline

12. Rosenkrantz AB, Geppert C, Grimm R, et al. Dynamic contrast-enhanced MRI of the prostate with high spatiotemporal resolution using compressed sensing, parallel imaging, and continuous golden-angle radial sampling: preliminary experience. J Magn Reson Imaging 2015;41:1365-73 CrossRef Medline

13. Wu X, Raz E, Block TK, et al. Contrast-enhanced radial 3D fatsuppressed T1-weighted gradient-recalled echo sequence versus conventional fat-suppressed contrast-enhanced T1-weighted studies of the head and neck. AJR Am J Roentgenol 2014;203:883-89 CrossRef Medline

14. Bangiyev L, Raz E, Block TK, et al. Evaluation of the orbit using contrast-enhanced radial 3D fat-suppressed $\mathrm{T} 1$ weighted gradient echo (Radial-VIBE) sequence. Br J Radiol 2015;88:20140863 CrossRef Medline

15. Heacock L, Gao Y, Heller SL, et al. Comparison of conventional DCE-MRI and a novel golden-angle radial multicoil compressed sensing method for the evaluation of breast lesion conspicuity. $J$ Magn Reson Imaging 2017;45:1746-52 CrossRef Medline

16. Rossi Espagnet MC, Bangiyev L, Haber M, et al. High-resolution DCE-MRI of the pituitary gland using radial k-space acquisition with compressed sensing reconstruction. AJNR Am J Neuroradiol 2015;36:1444-49 CrossRef Medline

17. Sen R, Sen C, Pack J, et al. Role of high-resolution dynamic contrastenhanced MRI with golden-angle radial sparse parallel reconstruction to identify the normal pituitary gland in patients with macroadenomas. AJNR Am J Neuroradiol 2017;38:1117-21 CrossRef Medline

18. Clopper CJ, Pearson ES. The use of confidence or fiducial limits illustrated in the case of the binomial. Biometrika 1934;26:404 CrossRef

19. Alibek S, Zenk J, Bozzato A, et al. The value of dynamic MRI studies in parotid tumors. Acad Radiol 2007;14:701-10 CrossRef Medline

20. Yabuuchi H, Matsuo Y, Kamitani T, et al. Parotid gland tumors: can addition of diffusion-weighted MR imaging to dynamic contrastenhanced MR imaging improve diagnostic accuracy in characterization? Radiology 2008;249:909-16 CrossRef Medline

21. Barrett T, Brechbiel M, Bernardo M, et al. MRI of tumor angiogenesis. J Magn Reson Imaging 2007;26:235-49 CrossRef Medline

22. Tao X, Yang G, Wang P, et al. The value of combining conventional, diffusion-weighted and dynamic contrast-enhanced MR imaging for the diagnosis of parotid gland tumours. Dentomaxillofac Radiol 2017:20160434 CrossRef Medline

23. Furukawa M, Parvathaneni U, Maravilla K, et al. Dynamic contrastenhanced MR perfusion imaging of head and neck tumors at $3 \mathrm{Te}-$ sla. Head Neck 2013;35:923-29 CrossRef Medline

24. Thomas AA, Arevalo-Perez J, Kaley T, et al. Dynamic contrast enhanced T1 MRI perfusion differentiates pseudoprogression from recurrent glioblastoma. J Neurooncol 2015;125:183-90 CrossRef Medline

25. Yuan Y, Tang W, Tao X. Parotid gland lesions: separate and combined diagnostic value of conventional MRI, diffusion-weighted imaging and dynamic contrast-enhanced MRI. Br J Radiol 2016;89: 20150912 CrossRef Medline

26. Gaddikeri S, Gaddikeri RS, Tailor T, et al. Dynamic contrast-enhanced MR imaging in head and neck cancer: techniques and clinical applications. AJNR Am J Neuroradiol 2016;37:588-95 CrossRef Medline 